\title{
The Effects of Single Session Forest Walking on Physiological and Psychological State of Myocardial Infarction Patients
}

\author{
Jung-Woo Shin ${ }^{1}$ and Jong-Hwan Choi ${ }^{2 *}$ \\ ${ }^{1}$ Graduate Department of Forest Therapy, Chungbuk National University, Cheongju 28644, Korea \\ ${ }^{2}$ Department of Physical Education, Chungbuk National University, Cheongju 28644, Korea
}

\begin{abstract}
Physical activities in the forest environment stimulate the parasympathetic nervous system of humans and have positive effects on the autonomic nervous system as well as moods and emotions. However, there are almost no studies on the benefits of exercise in the forest environment for patients with myocardial infarction. The aim of this study was to investigate the effects of 15-minute single session walking by myocardial infarction patients in the forest and urban environment on the physiological and psychological states. The heart rate variability was measured in 10 patients with myocardial infarction to assess physiological state after single session walking for 15 minutes in the forest environment and urban environment. In order to evaluate the psychological state, a profile of mood scale (POMS) and semantic differential (SD) questionnaire were used. The results of this study showed that 15-minute single session walking in the forest environment activated the parasympathetic nervous system of adult myocardial infarction patients more than 15 minutes of single session walking in the urban environment, and also made them feel more positive in terms of the POMS and SD. Therefore, this study suggests that even single session walking in the forest environment can positively influence the physiological and psychological states of adult heart disease patients and may also contribute to health care.
\end{abstract}

Keywords: autonomic nervous system, forest therapy, heart rate variability, profile of mood states, semantic differential method

\section{Introduction}

The incidence of myocardial infarction is increasing due to the westernized eating habits and smoking and drinking due to stress. Acute myocardial infarction is a disease in which coronary arteries that supply blood to the myocardium are suddenly blocked due to various causes, thereby resulting in myocardial necrosis (Korea Centers for Disease Control and Prevention, 2017). According to the announcement by WHO in 2015, heart disease is the leading cause of death in the world, killing 8.76 million (15.5\%) out of 5.4 million deaths in the world every year (World Health Organization, 2015). According to the Causes of Death Statistics in 2015 by Statistics Korea, 55.6 out of 100,000 people in the Korean population die from heart disease, accounting for $10.3 \%$ of total mortality rate and ranking second after cancer. Lack of physical activities and stress lower the activity of the parasympathetic nervous system (Thayer et al., 2010), and the activity of the 
sympathetic nervous system that increases relatively is more likely to lead to angina, temporary ischemia, fatal arrhythmia and sudden cardiac death (Lee et al., 1994). Moreover, Kleiger et al. (1987) stated that decrease in heart rate variability increases the risk of mortality by 5.3 times after myocardial infarction. In the early stage of acute myocardial infarction, there is a noticeable functional disturbance of the autonomic nervous system, which is slowly recovered after 6 months (Park et al., 1994).

Among various solutions to this problem, the forest environment may be a suitable alternative. Effects of the forest environment on the autonomic nervous system are as follows. Participants who took walks and mediated in the forest showed increase in the activity of the parasympathetic nervous system and decrease in the activity of the sympathetic nervous system after participating in the activities (Lee and Lee, 2012). It was also discovered from 10 college students that being in the valley reduced the activity of the sympathetic nervous system and increased the HF variable that represents the activity of the parasympathetic nervous system compared to being in the urban environment (Eom and Hwang, 2015). LF/HF that represents the balance of the sympathetic and parasympathetic nervous system decreased among women in their 50s after they took walks in the forest for 15 minutes (Lee and Shin, 2015).

Studies of effects on the mood and emotional states showed that in elementary school teachers, the experimental group that participated in the forest therapy program including forest walking showed decrease in tension-anxiety, depression, anger-hostility, confusion, fatigue, and total mood disturbance and increase in vigor compared to the control group that rested in daily life (Jo et al., 2015). As a result of applying the forest therapy program including forest walking to social welfare public officials and mental health workers, it was found that their tension-anxiety, depression, anger-hostility, confusion, fatigue, and total mood disturbance decreased whereas their vigor increased (Shin et al., 2015). Moreover, applying a yoga program in the healing forest to female adults resulted in the significant decrease of tension, depression, anger-hostility, fatigue, and confusion after the program (Park et al., 2017).

Most studies claimed that applying walking to normal adults in the forest environment was effective in regulating the autonomic nervous system and that it had positive effects on moods and emotions, but there was almost no research on the physiological and psychological effects of walking and exercising in the forest environment for myocardial infarction patients in Korea. Thus, the objective of this study is to assess the effects of walking in the forest and urban environments on the autonomous nervous system as well as mood and emotional states of myocardial infarction patients, and to determine a more suitable environment for physical activities.

\section{Research Methods}

\section{Subjects}

The subjects are 10 patients diagnosed with myocardial infarction and received percutaneous coronary intervention at C University Hospital located in C city, Chungbuk. Participants were specified as patients that received percutaneous coronary intervention at $\mathrm{C}$ University Hospital and were transferred to the department of rehabilitation medicine, who are aware of the research objectives and details of experiment and have agreed to participation. The subjects were those within 6 months of being diagnosed with myocardial infarction, and detailed characteristics are as shown in Table 1. Drinking, caffeine intake and smoking were prohibited before 8 hours as they may affect cardiovascular activity, and the subjects were to have enough sleep the night before in order to minimize fatigue on the day of the experiment. The research objectives and details of the experiment were described to the subjects and their consent for voluntary participation was obtained. This study was approved by the Institutional Review Board of Chungbuk National University Hospital. 


\section{Experimental Stimuli}

This study is on assessing physiological and psychological states due to single session walking in the forest and urban environments, and Gaesin Nanum (Sharing) Forest and a general rooftop was provided as stimuli for the experiment. Figure 1 shows images of the stimuli. At C University Hospital located in C city, a 350m-long circulation path at Gaesin Nanum (Sharing) Forest was selected as the forest environment, and the $180 \mathrm{~m}^{2}$ rooftop of C University Hospital surrounded in walls was selected as the urban environment. Gaesin Nanum (Sharing) Forest is a natural forest with oak (acorn, white oak, konara oak) with the clinical tree height of at least $20 \mathrm{~m}$, and the diameter at breast height of $25-30 \mathrm{~cm}$.

\section{Experimental method}

The participants were to do single session exercise walking for 15 minutes in the forest and urban environments. Before

Table 1. Characteristics of subjects patients

\begin{tabular}{lc}
\hline Subject $(\mathrm{N})$ & 10 \\
Male / female $(\mathrm{n})$ & $8 / 2$ \\
Age $($ year $)$ & $60.3 \pm 10.2$ \\
Height $(\mathrm{cm})$ & $165.12 \pm 6.5$ \\
Weight $(\mathrm{kg})$ & $66.63 \pm 10.4$ \\
Body mass index $\left(\mathrm{kg} / \mathrm{m}^{2}\right)$ & $24.33 \pm 2.7$ \\
Body fat percentage & $26.21 \pm 7.9$ \\
\hline
\end{tabular}

Note. $\mathrm{M} \pm \mathrm{SD}$.
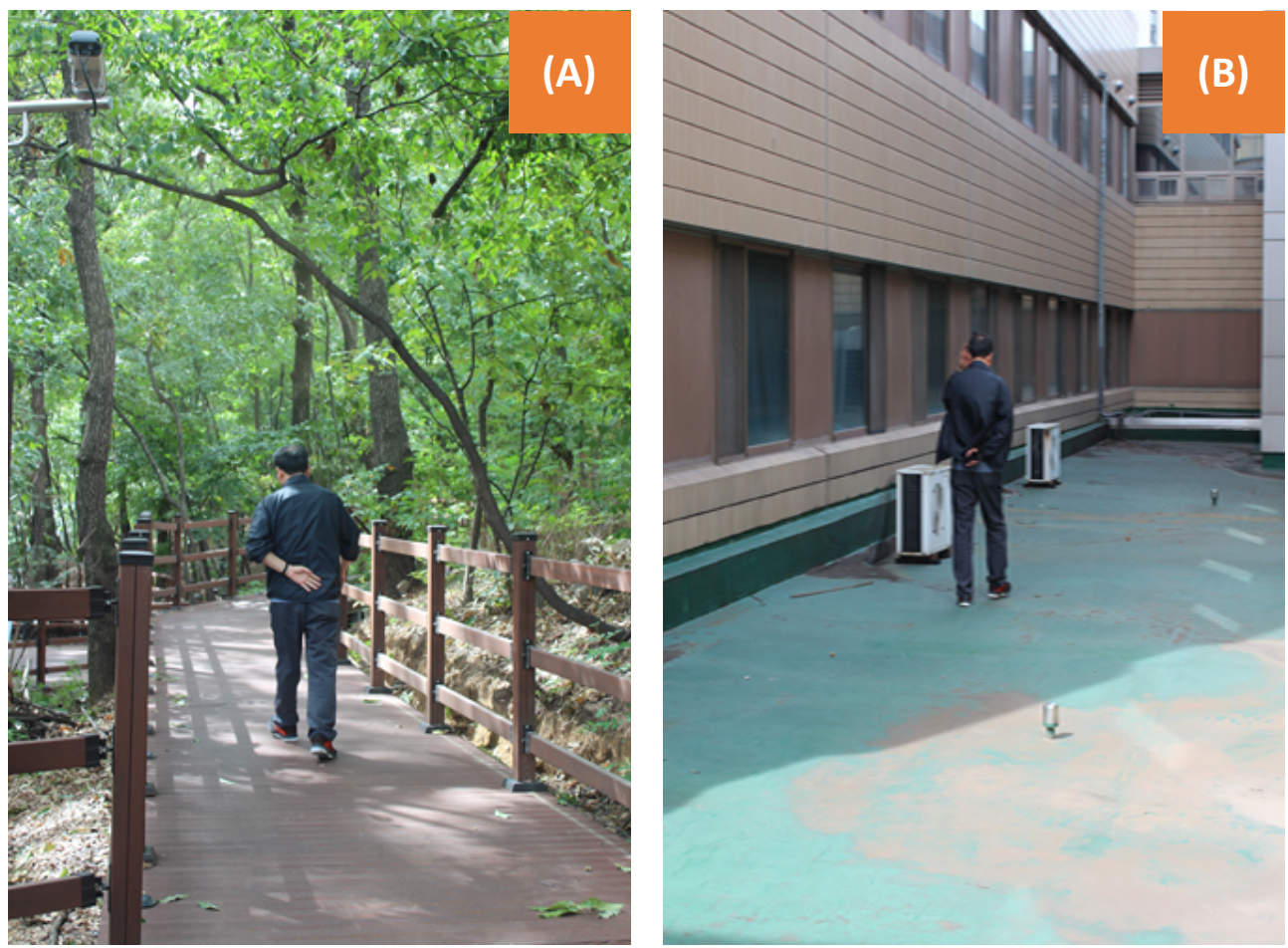

Figure 1. Images of experimental stilmuli at forest environment (A) and urban environment (B). 
participation, they listened to a brief explanation about the experiment and filled out a consent form for participation. They were randomly divided into two groups, with 5 participants in each group. Group A participated in single session walking for 15 minutes at their own pace around the circulation path of the Gaesin Nanum (Sharing) Forest. After that they had their heart rate variability (HRV) measured for 5 minutes in a closed $63 \mathrm{~m}^{3}$ space outside in order to measure their physiological response. After measuring HRV, they filled out the subjective questionnaires, Profile of Mood States (POMS) and Semantic Differential method (SD), about their psychological state in the same space. After that they rested for 30 minutes, and then repeatedly walked around the roof of the urban environment for 15 minutes at their own pace and performed the same assessment in the same space afterwards. On the contrary, Group B walked for 15 minutes in the urban environment. After that, the participants did the same assessment as Group A, and rested for 30 minutes. Then they did single session walking for 15 minutes in the forest environment and repeated the same assessment. The indexes measured after each single session walking were used to compare the physiological responses of the environments provided as the stimuli. Figure 2 shows the diagram of the experiment. To minimize exogenous variables, only one assistant participated in the place of experiment and measurement, and the place for the urban environment measurement was selected as a spot that is a minute away from the place of experiment with the same elevation. The temperature of the place of measurement was $26^{\circ} \mathrm{C}$, the humidity was $25 \%$, and the illumination was $281 \mathrm{Lux}$, all maintained consistently.

\section{Assessment tool}

To determine the effects on physiological and psychological states of heart disease patients in each environment, the subjects participated in the limited activity of walking for 15 minutes, and their HRV, mood states, and SD.

\section{Heart rate variability (HRV)}

HRV was used to measure physiological changes. HRV is an index reflecting the activity of the autonomic nervous system that is noninvasive and easy to measure, and it is widely used as the results can also be checked immediately after

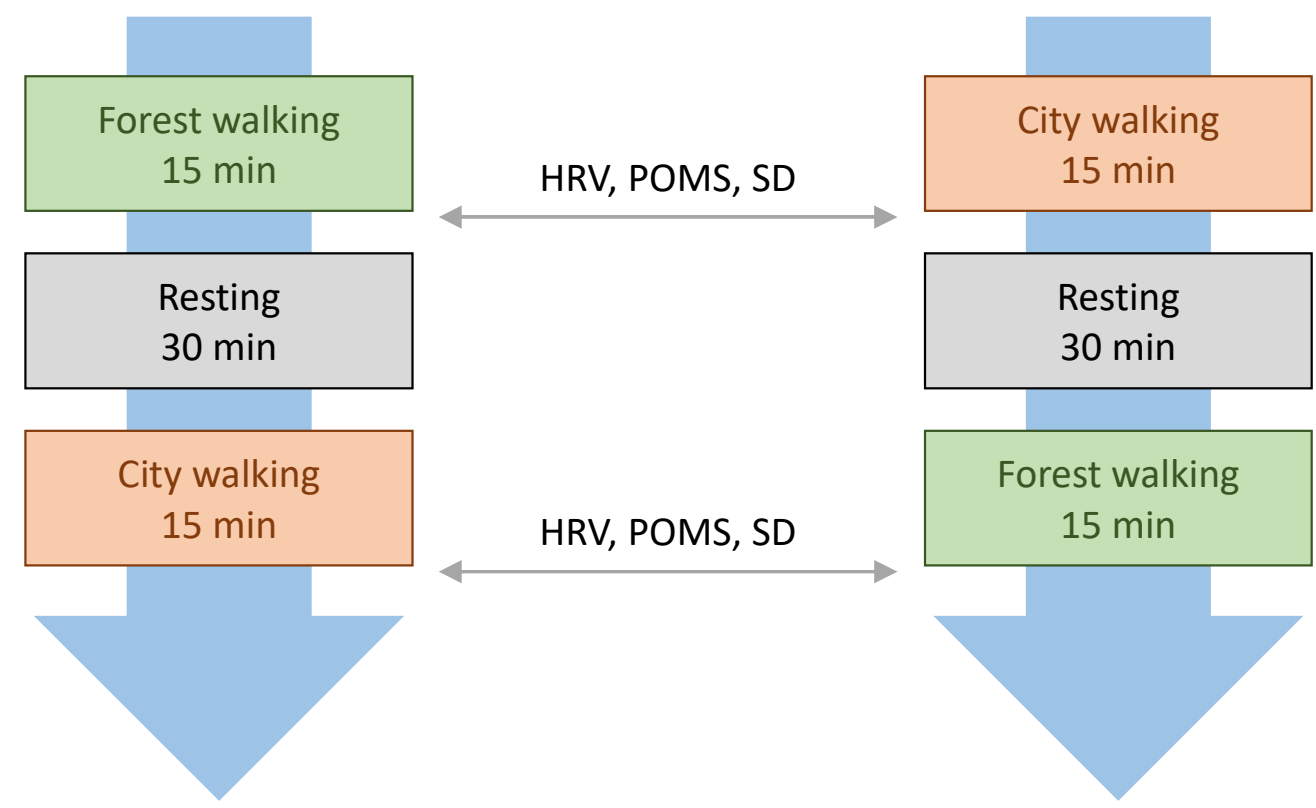

Figure 2. Experimental protocol. $\mathrm{HRV}=$ heart rate variability, $\mathrm{POMS}=$ profile of mood states, SD=Semantic differential method. 
measurement through computer analysis. Moreover, the results have high reproducibility and thus consistent results can be obtained from repeated measurements at consistent time intervals in the same conditions. This study used normHF (Normalized High Frequency) and LF/HF (Lower Frequency/High Frequency). Higher normHF is known to reflect more activity of the parasympathetic nervous system, and higher LF/HF reflects more activity of the sympathetic nervous system. HRV is measured using Canopy9 RSA of IEMBIO, and the measurement took 5 minutes in a relaxed state by lying down on a bed.

\section{Profile of Mood States (POMS)}

POMS is a questionnaire measuring human moods or emotions, designed to measure the current mood swings affected by the environment, and thus it is used to easily determine the effects of simple therapeutic intervention on moods. It consists of tension-anxiety, depression, anger-hostility, vigor, fatigue and confusion. Each item was rated on a 5-point Likert scale from 'Not at all (1 point)' to 'Very much (5 points). Total mood disturbance is the score subtracting the score of vigor from the sum of the scores of 5 sub-factors such as tension-anxiety, depression, anger-hostility, fatigue, and confusion. The survey was conducted after walking for 15 minutes in the forest and urban environments.

\section{Semantic Differential (SD) method}

The SD method evaluates the image space of humans based on emotional adjectives. This study used three adjectives such as comfortable and uncomfortable, natural and artificial, and calm and excited, which were rated on a 13-point scale from 1 point to 13 points. The survey was conducted after walking for 15 minutes in the forest and urban environments.

\section{Data analysis}

The data of this study were analyzed using the statistical program SPSS 21.0 (SPSS, IBM Company, USA). The mean (M) and standard deviation (SD) of physiological (HRV) and psychological stages (POMS, SD) after single session walking in the forest and urban environment were calculated, after which the Wilcoxon signed rank test was conducted to compare the differences. The statistical significance level was set at $p<.05$.

\section{Limitations}

The limitations of this study are as follows. First, the results were difficult to interpret and generalize due to the small sample size. The experiment was conducted on only a few myocardial infarction patients and thus could not control variables such as severity of the disease. Second, the changes of walking speed in each environment were not controlled, thereby creating a difference in the intensity of exercise. It was difficult to compare the results accurately as the variables according to the intensity of exercise were not controlled. Third, the experiment was conducted based on single session walking instead of a long-term exercise program.

\section{Results and Discussion}

This study was conducted on 10 myocardial infarction patients who participated in single session walking for 15 minutes in the forest environment (Gaesin Nanum (Sharing) Forest) and urban environment (general rooftop), in order to determine the effects of each environment on changes in HRV for physiological response and POMS and SD for psychological response of adult myocardial infarction patients. 


\section{Physiological response}

Table 2 shows the mean and standard deviation of HRV in the forest and urban environments after 15 minutes of single session walking. The norm HF reflecting the activity of the parasympathetic nervous system was $46.87 \pm 19.2$ after single session walking in the forest environment and $31.89 \pm 14.8$ after single session walking in the urban environment, indicating that the forest environment was significantly higher than the urban environment $(\mathrm{Z}=-2.803, p=.005)$. The $\mathrm{LF} / \mathrm{HF}$ ratio reflecting the overall balance between the sympathetic and parasympathetic nervous systems was $1.18 \pm 0.5$ after single session walking in the forest environment and $1.47 \pm 0.48$ after single session walking in the urban environment, indicating that the forest environment was significantly lower than the urban environment $(\mathrm{Z}=-2.666, p=.008)$.

\section{Psychological response}

Table 3 shows the mean and standard deviation of POMS in the forest and urban environments after 15 minutes of single session walking. As a result of the subjective evaluation POMS to measure six psychological states such as anger-hostility, confusion, depression, fatigue, tension-anxiety, vigor and total mood disturbance, there was a statistically significant difference in 5 items except for fatigue and vigor. Anger-hostility after single session walking in the forest was 4.9 \pm 4.7 , which was significantly lower than $11.8 \pm 8.8$ after single session walking in the urban environment. ( $Z=-2.347$, $p=.019)$. Moreover, confusion was 5.8 \pm 3.7 after single session walking in the forest environment, which was significantly lower than $9.4 \pm 4.2$ after single session walking in the urban environment $(\mathrm{Z}=-2.444, p=.015)$. Depression was $7.5 \pm 7.4$ after single session walking in the forest environment, which was also significantly lower than $15.9 \pm 1.3$ after single

Table 2. Result of heart rate variability after single session walking

\begin{tabular}{lcccc}
\hline \multirow{2}{*}{ Variable } & \multicolumn{3}{c}{$\mathrm{M} \pm \mathrm{SD}$} & \multirow{2}{*}{$\mathrm{Z}$} \\
\hline & Forest environment & Urban environment & & -2.803 \\
normHF (nu) & $46.87 \pm 19.20$ & $31.89 \pm 14.80$ & $.005^{* *}$ & $.008^{* *}$ \\
LF/HF & $1.18 \pm 0.50$ & $1.47 \pm 0.48$ & -2.666 \\
\hline
\end{tabular}

normHF=normalized high frequency; $\mathrm{LF} / \mathrm{HF}=$ low frequency/high frequency; nu=normalized unit.

${ }^{* * *}$ Significant at $p<.01$ by wilcoxon signed rank test.

Table 3. Result of POMS(Profile Of Mood States) after single session walking

\begin{tabular}{|c|c|c|c|c|}
\hline \multirow{2}{*}{ Variable } & \multicolumn{2}{|c|}{$\mathrm{M} \pm \mathrm{SD}$} & \multirow{2}{*}{$p$} & \multirow{2}{*}{$\mathrm{Z}$} \\
\hline & Forest environment & Urban environment & & \\
\hline Anger-hostility & $4.9 \pm 4.7$ & $11.8 \pm 8.8$ & $.019^{*}$ & -2.347 \\
\hline Confusion & $5.8 \pm 3.7$ & $9.4 \pm 4.2$ & $.015^{*}$ & -2.444 \\
\hline Depression & $7.5 \pm 7.4$ & $15.9 \pm 10.3$ & $.037^{*}$ & -2.090 \\
\hline Fatigue & $5.7 \pm 5.1$ & $9.6 \pm 6.6$ & .108 & -1.609 \\
\hline Tension-anxiety & $6.2 \pm 4.3$ & $11.9 \pm 6.1$ & $.022^{*}$ & -2.290 \\
\hline Vigour & $15.9 \pm 5.8$ & $11.5 \pm 5.3$ & .124 & -1.539 \\
\hline Total mood disturbance & $14.2 \pm 27.2$ & $47.1 \pm 36.8$ & $.028^{*}$ & -2.191 \\
\hline
\end{tabular}

\footnotetext{
** Significant at $p<.05$ by wilcoxon signed rank test.
} 
session walking in the urban environment $(\mathrm{Z}=-2.09, p=.037)$. Tension-anxiety was $6.2 \pm 4.3$ after single session walking in the forest environment, which was also significantly lower than $11.9 \pm 6.1$ after single session walking in the urban environment $(\mathrm{Z}=-2.29, p=.022)$. Moreover, fatigue was 5.7 \pm 5.1 after single session walking in the forest environment, which was lower than $9.6 \pm 6.6$ after single session walking in the urban environment, but there was no statistically significant difference $(\mathrm{Z}=-1.609, p=.108)$. Vigor was $15.9 \pm 5.8$ after single session walking in the forest environment, which was higher than $11.5 \pm 5.3$ after single session walking in the urban environment, but there was no statistically significant difference $(\mathrm{Z}=-1.539, p=.124)$. Total mood disturbance, which reflected all 6 mood states, was $14.2 \pm 27.2$ after single session walking in the forest environment, which was significantly lower than $47.1 \pm 36.8$ after single session walking in the urban environment $(\mathrm{Z}=-2.191, p=.028)$.

Table 4 shows the mean and standard deviation of the SD method in the forest and urban environments after 15 minutes of walking. The results of subjective assessment using the SD method showed a significant difference in all items of comfort, natural and calm. First, comfort was $12.3 \pm 0.7$ after single session walking in the forest environment, which was significantly higher than $6.1 \pm 4.3$ after single session walking in the urban environment $(\mathrm{Z}=-2.527, p=.012)$. Natural was $10.2 \pm 4.7$ after single session walking in the forest environment, which was significantly higher than $4.9 \pm 4.0$ after single session walking in the urban environment $(\mathrm{Z}=-2.371, p=.018)$. Moreover, calm was $11.9 \pm 2.5$ after single session walking in the forest environment, which was also significantly higher than $8.1 \pm 2.6$ after single session walking in the urban environment $(\mathrm{Z}=-2.501, p=.012)$.

The objective of this study is to determine the effects of applying the forest environment to adult heart disease patients on their physiological and psychological states. To this end, this study assessed the physiological and psychological states of the subjects after 15 minutes of single session walking in the forest and urban environments. HRV was used to assess the physiological response, and POMS and SD method were used to assess the psychological state, conducting a subjective survey.

First, the result of assessing the response of the autonomic nervous system showed that norm HF was significantly higher in the forest than the urban environment after 15 minutes of single session walking, and LF/HF was significantly lower in the forest than the urban environment. This result is consistent with previous studies proving that the parasympathetic nervous system is activated in the forest environment and the sympathetic nervous system is suppressed (Tsunetsugu et al., 2007; Park et al., 2010; Lee et al., 2014). Park et al. (2010) reported that even in the same forest environment, the activity of the parasympathetic nervous system was higher in walking than enjoying landscape. This difference implies that various factors of the forest environment such as phytoncide, anion, landscape, sunlight and topography have effect, these factors also require dynamic activities in order to have greater effect on the subjects. A study divided participants into a group with high physical fitness and a group with low physical fitness according to the scores of

Table 4. Result of SD(Semantic Differential method) after single session walking

\begin{tabular}{lcccc}
\hline \multirow{2}{*}{ Variable } & \multicolumn{3}{c}{$\mathrm{M} \pm \mathrm{SD}$} & \multicolumn{2}{c}{$\mathrm{Z}$} \\
\hline & Forest environment & Urban environment & $.012^{*}$ & -2.527 \\
Comfort & $12.3 \pm 0.7$ & $6.1 \pm 4.3$ & $.018^{*}$ & -2.371 \\
Natural & $10.2 \pm 4.7$ & $4.9 \pm 4.0$ & $.012^{*}$ & -2.501 \\
Calm & $11.9 \pm 2.5$ & $8.1 \pm 2.6$ & \\
\hline
\end{tabular}

Note. Each item was rated on a 13-point scale.

${ }^{* *}$ Significant at $p<.05$ by wilcoxon signed rank test. 
the University of Houston Non-Exercise Test, and proved that the group with high physical fitness was superior in controlling the parasympathetic nervous system (Rossy and Thayer, 1998). Rennie et al. (2003) and Chandola et al. (2008) claimed that the parasympathetic nervous system is activated and the sympathetic nervous system is suppressed in the group of public officials with $25 \%$ of high intensity physical activities. According to Sloan et al. (2009) and Lee et al. (2011) who conducted research through experimental intervention, the activity of the parasympathetic nerve index increased with 12 weeks of aerobic exercise, but Melville et al. (2012) and Cheema et al. (2013) stated that there was no significant change in the parasympathetic nerve index in frequency after applying 15 minutes of yoga and meditation, and 50 minutes of yoga 3 times a week for 10 weeks. The increased activity of the sympathetic nervous system and decreased activity of the parasympathetic nervous system are related to increasing the risk of cardiac death and ventricular arrhythmia (Lahiri et al., 2008), and that lack of physical activities, which is a crucial risk factor of heart disease, is correlated with reducing the activity of the parasympathetic nervous system and increasing resting heart rate (Thayer et al., 2010). Considering that, the forest environment is more desirable than the urban environment for activating the parasympathetic nervous system, and it is more important to increase physical activities such as walking or aerobic exercise than static activities like yoga, meditation or watching the view in the forest environment.

Second, the result of assessing psychological state showed that anger-hostility, confusion, depression, tension-anxiety, and total mood disturbance was significantly lower in the forest than the urban environment after 15 minutes of single session walking, while fatigue and vigor each showed a decrease and increase but had no significance. This result is partially consistent with previous research on college students proving that walking for 15 minutes in the forest of 24 regions decreased the scores of anger-hostility, confusion, depression, tension-anxiety, fatigue and total mood disturbance and increased the score of vigor more than the urban environment (Park et al., 2010). However, there were other results as well. A study was conducted on 72 workers divided into groups who were to look at the forest on the rooftop and the outdoor parking lot for 5 minutes. Male participants showed a significant increase in vigor and significant decrease in fatigue on the rooftop, while female participants showed a significant decrease in tension-anxiety, depression and fatigue and a significant increase in vigor (Matsunaga et al., 2011). There was also a study on young adults who were to walk for 12 minutes in the forest and urban area, and then switched places the next day, which proved that except depression, all items such as anger-hostility, confusion, fatigue, tension-anxiety, vigor and total mood disturbance showed a significant difference (Lee et al., 2014). When applying a yoga program using the healing forest to female adults, the scores of tension-anxiety, depression, anger-hostility, fatigue, and confusion significantly decreased after the program (Park et al., 2017). The results of previous studies prove that there are more effects on the subjects when they participate actively in programs rather than passively enjoying the view, and that the effects vary depending on the quality and quantity of the forest. Thus, enthusiastic activities in an abundant forest are effective in POMS.

Third, changes in comfort, natural and calm after 15 minutes of single session walking were significantly higher in the forest than the urban environment. This result is consistent with the study claiming that the scores of comfort, natural, and calm increased after 15 minutes of forest walking (Lee et al., 2014). Moreover, Ochiai et al. (2015) conducted a study on middle-aged women in Japan and reported that the scores of comfort, natural, and calm were significantly higher after 4 hours of the forest therapy program than before. Joung et al. (2015) conducted a study on 8 college students and claimed that watching the view of the forest from the rooftop of a 4-story building in the fall led to significantly higher comfort, natural and calm than watching the view of the city. Song et al. (2017) had 20 middle-aged men with hypertension watch the view of the forest and the city, and proved that the forest landscape showed significantly higher scores of comfort, natural and calm than the urban landscape. Thus, it is proved that the forest environment induces higher emotional stability than the urban environment. 
In sum, 15 minutes of single session walking in the forest environment for adult heart disease patients positively improved HRV, stabilized the autonomic nervous system, and improved mood and emotional states. Follow-up research need to conduct long-term physiological and psychological evaluation of heart disease patients instead of a single session through a program with consistent exercise intensity, time and frequency that can generalize the number and gender of subjects. Nonetheless, this study suggests that even single session walking in the forest environment positively affects physiological and psychological states of adult heart disease patients and even contributes to their health care.

\section{Conclusion}

Physical activities in the forest environment stimulate the parasympathetic nervous system of humans and have positive effects on the autonomic nervous system as well as moods and emotions. However, there is almost no research on the benefits of exercise in the forest environment for myocardial infarction patients. The objective of this study is to examine the effects of 15-minute single session walking by myocardial infarction patients in the forest and urban environments on their physiological and psychological states. This study had 10 myocardial infarction patients participate in 15 minutes of single session walking in the forest and urban environments, after which their HRV was measured to assess their physiological state, and POMS and SD method were conducted to assess their psychological state. The results showed that 15 minutes of single session walking in the forest environment activated the parasympathetic nervous system of adult myocardial infarction patients more and made them feel more positive in the POMS and SD items compared to 15 minutes of single session walking in the urban environment. Therefore, this study suggests that even single session walking in the forest environment positively affects physiological and psychological states of adult heart disease patients and even contributes to their health care.

\section{References}

Chandola, T., A. Britton, E. Brunner, H. Hemingway, M. Malik, M. Kumari, E. Badrick, M. Kivimaki, and M. Marmot. 2008. Work stress and coronary heart disease: what are the mechanisms? Eur. Heart J. 29(5):640-648. DOI:10.1093/eurheartj/ehm584

Cheema, B.S., A. Houridis, L. Busch, V. Raschke-Cheema, G.W. Melville, P.W. Marshall, D. Chang, B. Machliss, C. Lonsdale, J. Bowman, and B. Colagiuri. 2013. Effect of an office worksite-based yoga program on heart rate variability: outcomes of a randomized controlled trial. BMC Complement. Altern. Med. 13:82.

DOI:10.1186/1472-6882-13-82

Eom, P.D. and M.C. Hwang. 2015. Effects of viewing environments of valley, forest road, and city on emotional state based on autonomic nervous system. J. Korean Inst. For. Recreat. 19(4):1-12.

Jo, M.N., C.S. Shin, P.S. Yeoun, and J.Y. Kim. 2015. The effects of the forest healing program according to job-stress, fatigue, mood state of the elementary school teachers. Proc. J. Korean Inst. For. Recreat. 2015.4:408.

Joung, D.W., G.W. Kim, Y.H. Choi, H.J. Lim, S.J. Park, J.M. Woo, and B.J. Park. 2015. The prefrontal cortex activity and psychological effects of viewing forest landscape in autumn season. Int. J. Environ. Res. Public Health 12(7):72357243. DOI:10.3390/ijerph120707235

Kleiger, R.E., J.P. Miller, J.T. Bigger Jr, A.J. Moss. 1987. Decreased heart rate variability and its association with increased mortality after myocardial infarction. Am. J. Cardiol. 59(4):256-262. DOI:10.1016/0002-9149(87)90795-8

Korea Centers for Disease Control and Prevention (KCDC). 2017. Retrieved fromhttp://health.mw.go.kr

Lahiri, M.K., P.J. Kannankeril, and J.J. Goldberger. 2008. Assessment of autonomic function in cardiovascualr disease: physiological basis and prognostic implications. J. Am. Coll. Cardiol. 51(18):1725-1733. DOI:10.1016/j.jacc.2008.01.038

Lee, B.G. and H.H. Lee. 2012. A study on the effects of human physiology after forest phytoncide therapy. J. Naturopathy 
$1(1): 14-20$.

Lee, H.H., I.G. Jeong, M.J. Oh, S.Y. Yoon, and B.Y. Lee. 2011. Effect of 12-weeks exercise program on heart rate variability in middle-aged obese woman. J. Sport Leis. Stud. 43(2):833-842.

Lee, J.Y., Y. Tsunetsugu, N. Takyama, B.J. Park, Q. Li, C.R. Song, M. Komatsu, H. Ikei, L. Tyraväinen, T. Kagawa, and Y. Miyazaki. 2014. Influence of forest therapy on cardiovascular relaxation in young adults. Evid. Based Complement. Alternat. Med. 2014(article ID 834360):1-7. DOI:10.1155/2014/834360

Lee, T.I., K.W. Choi, Y.J. Kim, D.G. Shin, Y.J. Kim, B.S. Shim, H.W. Lee, and K.H. Lee. 1994. Effect of stress on cardiovascular autonomic nervous activity in recovering myocardial infarction patients and normal controls measured by power spectral analysis. Korean Circ. J. 24(1):24-37. DOI:10.4070/kcj.1994.24.1.24

Lee, Y.J. and C.S. Shin. 2015. Influence of forest healing user's nature relatedness, emotion, arousal state on stress recovery - Focused on women in theirs 50's -. J. Korean Soc. People Plants Environ. 18(2):123-128.

Matsunaga, K., B.J. Park, and Y. Miyazaki. 2011. Determination of subjective relaxation effects of a hospital rooftop forest on healthcare workers - using POMS and STAI-FormJYZ-. J. Japanese Soc. Balneol. Climatol. Phys. Med. 74(3):186-199. DOI:10.11390/oniki.74.186

Melville, G.W., D. Chang, B. Colagiuri, P.W. Marshall, and B.S. Cheema. 2012. Fifteen minutes of chair-based yoga postures or guided meditation performed in the office can elicit a relaxation response. Evid. Based Complement. Alternat. Med. 2012(article ID 501986):1-9. DOI:10.1155/2012/501986

Ochiai, H., H. Ikei, C. Song, M. Kobayashi, T. Miura, T. Kagawa, Q. Li, S. Kumeda, M. Imai, and Y. Miyazaki. 2015. Physiological and Psychological effects of a forest therapy program on middle-aged females. Int. J. Environ. Res. Public Health 12(12):15222-15232. DOI:10.3390/ijerph121214984

Park, B.J., Y. Tsunetsugu, T. Kasetani, T. Kagawa, and Y. Miyazaki. 2010. The physiological effects of shinrin-yoku (taking in the forest atmosphere or forest bathing): evidence from field experiments in 24 forests across Japan. Environ. Health Prev. Med. 15(1):18-26. DOI:10.1007/s12199-009-0086-9

Park, C.E., D.J. Kim, C.S. Shin, and Y.H. Kim. 2017. Effects of yoga program using healing forest space on mood and psychological well-being of adult women. Proc. J. People Plants Environ. 6:105.

Park, J.W., H.J. Youn, W.S. Chung, J.C. Park, C.M. Kim, I.S. Park, J.H. Kim, K.B. Choi, and S.J. Hong. 1994. Changes of responses of autonomic nervous system in patients after myocardial infarction. Korean Circ. J. 24(2):272-279.

Rennie, K.L., H. Hemingway, M. Kumari, E. Brunner, M. Malik, and M. Marmot. 2003. Effects of moderate and vigorous physical activity on heart rate variability in a British study of civil servants. Am. J. Epidemiol. 158(2):135-143. DOI:10.1093/aje/kwg120

Rossy, L.A. and J.F. Thayer. 1998. Fitness and gender-related differences in heart period variability. Psychosom. Med. 60(6):773-781.

Shin, C.S., P.S. Yeoun, Y.G. Kim, J.O. Eum, Y.R. Yim, S.B. Yoon, S.H. Park, I.O. Kim, and S.H. Lee. 2015. The influence of a forest healing program on public servants in charge of social welfare and mental health care worker's job stress and the profile of mood states(POMS). J. Korean For. Soc. 104(2):294-299. DOI:10.14578/jkfs.2015.104.2.294

Sloan, R.P., P.A. Shapiro, R.E. DeMeersman, E. Bagiella, E.N. Brondolo, P.S. Mckinley, I. Slavov, Y. Fang, and M.M. Myers. 2009. The effect of aerobic training and cardiac autonomic regulation in young adults. Am. J. Public Health 99(5):921-928. DOI:10.2105/AJPH.2007.133165

Song, C., H. Ikei, M. Kobayashi, T. Miura, Q. Li, T. Kagawa, S. Kumeda, M. Imai, and Y. Miyazaki. 2017. Effect of viewing forest landscape on middle-aged hypertensive men. Urban For. Urban Green. 21:247-252. DOI:10.1016/j.ufug.2016.12.010

Thayer, J.F., S.S. Yamamoto, and J.F. Brosschot. 2010. The relationship of autonomic imbalance, heart rate variability and cardiovascular disease risk factors. Int. J. Cardiol. 141(2):122-131. DOI:10.1016/j.ijcard.2009.09.543

Tsunetsugu, Y., B.J. Park, H. Ishii, H. Hirano, T. Kagawa, and Y. Miyazaki. 2007. Physiological effects of shinrin-yoku (taking in the atmosphere of the forest) in an old-growth broadleaf forest in Yamagata prefecture, Japan. J. Physiol. Anthropol. 26(2):135-142. DOI:10.2114/jpa2.26.135

World Health Organization(WHO). 2015. Retrieved from http://www.who.int 